\title{
Distributed Top-N Query Processing with Possibly Uncooperative Local Systems
}

\author{
Clement Yu, George Philip \\ Dept. of Computer Science \\ U. of Illinois at Chicago \\ Chicago, IL 60607 \\ \{yu,gphilip\}@cs.uic.edu
}

\author{
Weiyi Meng \\ Dept. of Computer Science \\ SUNY at Binghamton \\ Binghamton, NY 13902 \\ meng@cs.binghamton.edu
}

\begin{abstract}
We consider the problem of processing top-N queries in a distributed environment with possibly uncooperative local database systems. For a given top- $\mathrm{N}$ query, the problem is to find the $\mathrm{N}$ tuples that satisfy the query the best but not necessarily completely in an efficient manner. Top- $\mathrm{N}$ queries are gaining popularity in relational databases and are expected to be very useful for e-commerce applications. Many companies provide the same type of goods and services to the public on the Web, and relational databases may be employed to manage the data. It is not feasible for a user to query a large number of databases. It is therefore desirable to provide a facility where a user query is accepted at some site, suitable tuples from appropriate sites are retrieved and the results are merged and then presented to the user. In this paper, we present a method for constructing the desired facility. Our method consists of two steps. The first step determines which databases are likely to contain the desired tuples for a given query so that the databases can be ranked based on their desirability with respect to the query. Four different techniques are introduced for this step with one requiring no cooperation from local systems. The second step determines how the ranked databases should be searched
\end{abstract}

Permission to copy without fee all or part of this material is granted provided that the copies are not made or distributed for direct commercial advantage, the VLDB copyright notice and the title of the publication and its date appear, and notice is given that copying is by permission of the Very Large Data Base Endowment. To copy otherwise, or to republish, requires a fee and/or special permission from the Endowment.

Proceedings of the 29th VLDB Conference, Berlin, Germany, 2003 and what tuples from the searched databases should be returned. A new algorithm is proposed for this purpose. Experimental results are presented to compare different methods and very promising results are obtained using the method that requires no cooperation from local databases.

keywords: Top-N queries, distributed databases, query processing.

\section{Introduction}

As pointed out in $[1,3,4,6,7]$, it is of great interest to find the $N$ tuples in a database table which best match a given user query, for some integer $N$. If the table contains tuples that describe cars, then the problem becomes finding the $N$ best matching cars of a given car description. Current commercial relational database systems do not support the processing of such type of queries. Techniques for processing such queries in a centralized environment have recently been proposed by $[1,3,4,6]$. In this paper, we examine the processing of this type of queries in large-scale distributed relational databases. Specifically, given a query that requests the top $N$ matched tuples (or top $N$ tuples for short) across many databases, we study different methods to determine the databases that are likely to contain the desired results. This is of special interest in the Internet environment where numerous sites may provide data about the same type of products/services. In such an environment, most users will be satisfied with getting most of the top $N$ results.

A straightforward way to process a top-N query in a distributed environment is to send the query to all databases. The site of each database returns the top $N$ local tuples, which are then merged with results from other sites at a common site to produce the overall top $N$ tuples. This strategy is, however, not efficient if the number of databases is large, because most of them 
probably won't contain any of the desired tuples. For example, if $N=20$ and the number of databases is 200 , then at least 180 of the databases won't be useful for this query. This simple method incurs unnecessary cost to send the query to the useless sites and unnecessary cost to process the query at these sites. Furthermore, when these sites return their retrieval results to the common site, there will be further waste of communication and local processing resources.

In this paper, we present a two-step process to find the top $N$ matched tuples of a given relational query in a distributed database environment. In the first step, an attempt is made to rank databases optimally with respect to the given query based on certain desirability criterion. Four different histogram based methods are utilized to estimate the desirability of each database with respect to the query. In the second step, a merge algorithm is used to determine which databases should be accessed and what tuples from accessed databases should be retrieved. Retrieved tuples are merged to form the output to the user. A new merge algorithm is proposed in this paper. Experimental results are carried out to compare the performance, in terms of both accuracy (effectiveness) and efficiency, of different approaches. The contributions of this paper are:

1. In the e-commerce environment, it is likely that many databases (owned by different companies) are not willing to supply sufficient data for the construction of the histograms. We propose a method to construct histograms from uncooperative databases and we demonstrate that its ability in retrieving the top $N$ tuples is significantly higher than other histogram construction techniques. To the best of our knowledge, this is the first paper on the top-N query problem in distributed uncooperative relational databases.

2. We provide a new merge algorithm and compare it both analytically and experimentally with an existing merge algorithm. It is found that the new algorithm has higher retrieval effectiveness but lower efficiency.

3. Various histogram construction and estimation techniques are compared. Experimental results indicate that our proposed histogram construction algorithm that requires no cooperation from databases are much more accurate than three other histogram generation algorithms. Furthermore, the new algorithm gives consistently high accuracies under different query types (different dimensions, different distance functions, or a mixture of various query types).

4. We identify the type of queries where the original merge algorithm is sufficiently accurate and the type of queries where the new merge algorithm is needed for high accuracy. As a consequence, we utilize both merge algorithms to achieve both high accuracy and high efficiency.

A comparison with related literature is as follows.

1. The work reported in this paper can be consid- ered as a generalization of the top- $\mathrm{N}$ query problem from centralized environments to distributed environments. The problem here is to identify the independently operated databases that are likely to contain the top $N$ tuples for a given query; such a problem does not exist in a centralized environment.

2 . Some recent techniques to construct histograms $([5,9,11,13,18,19])$ are employed here, although there is a significant difference. Histograms were traditionally used to estimate the number of tuples satisfying a certain query condition. In this paper, we modify existing techniques and propose a new technique so that they can be used to estimate the distance of the best matched tuple in a database to a given query.

3. Database (source) selection techniques discussed in $[16,17]$ do not consider top- $\mathrm{N}$ queries.

4. Preference queries have received some attention recently [10, 12]. While preference queries support richer mechanisms for a user to express preference of results than top-N queries, the evaluation techniques for these two types of queries are very different. Top$\mathrm{N}$ query processing aims to evaluate only tuples that have the potential to be ranked among the top $\mathrm{N}$ results and uses histogram-based techniques to narrow the search space. Preference query evaluation typically ranks all tuples and uses views or materialized views to support preference capabilities [10, 12].

5 . The most closely related work to this paper is [23] but there are several significant differences between it and this paper. First, a new merge algorithm is proposed, which is is compared with that in [23] in this paper. Second, [23] used just one type of histograms to estimate the distance of the best matched tuple in a database and no comparison with other methods was made. In this paper, three different types of histograms are utilized and compared with the one used in [23]. Third, the estimation algorithm in [23] is of exponential complexity, while the estimation algorithm in this paper takes low polynomial time complexity. Fourth, the new merge algorithm and the original merge algorithm are utilized jointly to achieve high accuracy and efficiency.

\section{Examples of Top-N Queries}

In this section, we provide a few examples to illustrate the use of the top- $N$ queries. While such queries are widely used in text and multimedia databases, they are rather unusual in relational databases but are gaining importance. Their interpretations are by no means standard and are application dependent. Due to their different interpretations and applicabilities, we classify these queries into three different types. We also provide "distance" functions which may be suitable for the three different situations.

\section{(a) Standard top-N queries}

Given a relation $R\left(A_{1}, \ldots, A_{m}\right)$, where the A's are the attributes of the relation and a query $Q\left(q_{1}, \ldots, q_{m}\right)$, 
where $q_{i}$ is a condition on attribute $A_{i}, i=1, \ldots, m$, a distance function $d$ such as the Euclidean distance or the Manhattan distance can be defined such that a distance $d(Q, t)$ can be computed, where $t=\left(t_{1}, \ldots, t_{m}\right)$ is a tuple in $R$. The distance is a measure of how well the tuple $t$ satisfies the query $Q$.

Example 1 Consider a relation about used cars that includes attributes price, $p$, and mileage, $m$. Suppose a user is interested in finding a used car satisfying his/her requirements on these two attributes. Suppose the user submits the following SQL query.

Select C.id, C.p, C.m From Used-car C

Where C.p $=2000$ and C.m $=100000$

There may not be a tuple satisfying the given conditions. The remedy is to have a distance function $f$ such that a distance can be computed between the query, $Q(p=2000, m=100000)$ and each tuple $t$. Then the tuples are ordered in ascending order of distances. Finally, the $N$ tuples having the smallest distances are presented to the user, where $N$ is an integer specified by the user. This may be indicated by the SQL-like query below:

Select C.id, C.p, C.m (10) From User-car C

Where C.p $=2000$ and C.m $=100000$

Order by Distance $f$

Here, the 10 best matched tuples are to be given to the user, where the distance function is $f$.

Although the above example is reasonable, there are rooms for better interpretations. First, if a car has an additional 10,000 miles, it may still fit the user's need. But, if the car costs an additional $\$ 10,000$, it will definitely not be suitable to the user. This can be remedied by normalizing the attribute value of each tuple by the corresponding query attribute value for each attribute. For example, if a tuple has the price and the mileage given by $(2300,110,000)$, then the percentage differences in the two attributes are $(300 / 2000,10,000 / 100,000)=(15 \%, 10 \%)$. For the rest of this paper, we shall use the percentage difference instead of the absolute difference. Often, different attributes may have different degrees of significance to the user. An importance factor $I_{i}$ can be associated with the ith attribute.

If the Manhattan distance is used, the "distance" due to the $i$-th attribute, $d_{i}$, is given by $\left(\left|t_{i}-q_{i}\right| / q_{i}\right) * I_{i}$ and the overall distance due to multiple attributes is $\sum_{i}\left(\left|t_{i}-q_{i}\right| / q_{i}\right) * I_{i}$. If the Euclidean distance function is used, then $d_{i}=\left(\left(t_{i}-q_{i}\right) / q_{i}\right)^{2} * I_{i}$ and the overall distance is $\sqrt{\sum_{i}\left(\left(t_{i}-q_{i}\right) / q_{i}\right)^{2} * I_{i}}$.

\section{(b) Generalized "distance" top-N queries}

In the above case, a car costing $\$ 1,500$ is at the same distance as another car costing $\$ 2,500$ relative to the query condition of $\$ 2,000$, though the former car with the same mileage as the latter is likely to be more desirable to the user. To achieve the desired effect, the "distance" function is adjusted to permit negative values. In deciding the top $N$ tuples of a query, the tuples are sorted in ascending order of distance, with negative distances ahead of positive ones.

If the Manhattan distance is used, $d_{i}=\left(\left|t_{i}-q_{i}\right| / q_{i}\right) *$ $I_{i}$, if $t_{i} \geq q_{i}$; otherwise, $d_{i}=-\left(\left|t_{i}-q_{i}\right| / q_{i}\right) * I_{i}$. If the Euclidean distance is used, then $d_{i}=\left(\left(t_{i}-q_{i}\right) / q_{i}\right)^{2} * I_{i}$, if $t_{i} \geq q_{i}$; otherwise, $d_{i}=-\left(\left(t_{i}-q_{i}\right) / q_{i}\right)^{2} * I_{i}$. Again, if the Euclidean distance is used and when all attributes are considered, the distances due to the individual attributes are summed and then the square root is taken. If a summed "distance" is negative, the absolute value is taken before the square root is performed and the negative sign is then added back in.

\section{(c) Two sided generalized "distance" top-N queries}

Suppose we are interested in seeking an airplane ticket from Chicago to New York City. The attributes of interest could be the price and the time of departure. For the time of departure, we may specify a range of time which is acceptable, for example from $3 \mathrm{pm}$ to $5 \mathrm{pm}$. Any time within the range will incur a distance of 0 , but a time outside the range incurs a positive distance. As indicated before, we are interested in the percentage difference in each attribute. Thus, the denominator of the distance function for normalization due to time is set to be the mid-point, i.e., $4 \mathrm{pm}$ in the above example. This is to ensure that one hour deviation from either side outside the range incurs the same distance. For example, a 2pm departure time incurs a percentage distance of $1 / 4$. Recall that for each attribute there is an importance factor. This can be set to eliminate the effect of where the mid-point lies. For example, the importance factor can be set to be $I_{i} * m_{i}$, where $m_{i}$ is the mid-point of the interval (equal to 4 in the above example), and $I_{i}$ is the relative importance of the ith attribute. With these parameter values, the mid-point $m_{i}$ will be cancelled out from both the numerator and the denominator.

Let a range $\left(l_{i}, u_{i}\right)$ be specified for the ith-attribute. Let $m_{i}$ be the mid-point in the range, i.e., $\left(u_{i}-l_{i}\right) / 2$. If the Manhattan distance is used,

$$
d_{i}= \begin{cases}\left|t_{i}-u_{i}\right| / m_{i} * I_{i}, & \text { if } t_{i}>u_{i} \\ \left|l_{i}-t_{i}\right| / m_{i} * I_{i}, & \text { if } l_{i}>t_{i} \\ 0, & \text { if } t_{i} \text { is in }\left(l_{i}, u_{i}\right)\end{cases}
$$

If the Euclidean distance is used,

$$
d_{i}= \begin{cases}\left(\left(t_{i}-u_{i}\right) / m_{i}\right)^{2} * I_{i}, & \text { if } t_{i}>u_{i} \\ \left(\left(l_{i}-t_{i}\right) / m_{i}\right)^{2} * I_{i}, & \text { if } l_{i}>t_{i} \\ 0, & \text { if } t_{i} \text { is in }\left(l_{i}, u_{i}\right)\end{cases}
$$

For the rest of this paper, we shall concentrate on these three types of top-N queries. For each type, we shall utilize the "Euclidean distance" function and the "Manhattan distance" function (for generalized distance top-N queries, negative "distance" may arise.)

The use of the top- $N$ queries arises naturally in ecommerce applications where the specification of desired products is more complex. For example, when 
buying long term care insurance for a couple, numerous criterions may be significant. They may include the number of years requiring long term care, the way the number of years are split between the couple (say the older person gets more years than the younger person), the amount of expenses allowed per day, the amount of deductible, whether the amount of allowable expenses is adjusted based on inflation, the extent the amount is applied to home care versus nursing home, and the amount the couple can afford for premium per year. It is very likely that not all of these criterions can be satisfied simultaneously. As a result, the best matches to the specification are needed.

\section{Deciding Databases to Search}

Our aim is to retrieve the the $N$ tuples with the smallest distances from the query. This needs to be done in a distributed environment across many databases. One site may be designated as a global site which assembles the tuples and returns them to the user. This site has a global distance function which is the one desired by the user. In this paper, we assume that there is one relation in each database. Each database is operated autonomously at a different site. For the remaining part of this paper, "relation" and "database" will be used interchangeably.

To facilitate the identification of the top $N$ tuples for a given query, we store for each relation a "histogram", taking into consideration some dependencies between the attributes. The histograms for all sites are stored at the global site where user queries are processed. They are used to rank databases in descending order of estimated desirability with respect to each query, based on the global distance function. Tuples from the selected databases are retrieved in such a way that if the databases are ranked optimally, then all the top $N$ tuples will be retrieved.

In Section 3.1, we describe the constructions of four types of histograms that will be used to estimate the desirability of each database with respect to any given query. In Section 3.2, a method for ranking databases optimally with respect to a given query is provided. The method is to simply rank databases in ascending order of the distance of the best matched tuple in each database. In Section 3.3, algorithms are given to estimate the distance of the best matched tuple in each database to the query based on different types of histograms. In Section 3.4, a new merge algorithm is provided to determine the tuples to be retrieved from the ranked databases. This algorithm is then compared with a previous merge algorithm [23] analytically.

\subsection{Histogram Construction}

In this section, the constructions of four different types of histograms are presented. Due to the space limitation, only two types will be described in some detail.

\section{(A) Linear Approximation (LA)}

The construction of this type of histogram will be given as follows and will be illustrated by the used-car relation $R$ on price and mileage. The general case will be sketched later in Section 3.3.

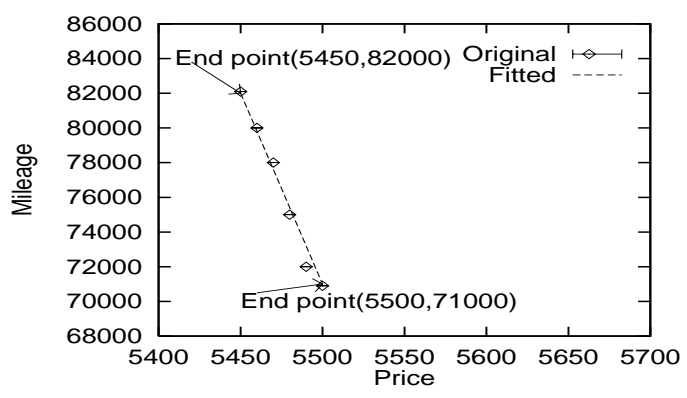

Figure 1: Linear Approximation of a Price Interval

(1). The domain of the price attribute is partitioned into fixed size intervals (each interval has a range of $\$ 50)$ as follows: \begin{tabular}{|l|l|l|l|l|l|}
\hline Price & $\ldots$ & $\$ 5400$ & $\$ 5450$ & $\$ 5500$ & $\ldots$ \\
\hline
\end{tabular}

(2). All the tuples within an interval are replaced by a straight line, which minimizes the least square error. For example in Figure 1, the line is a least square approximation to the various points in the interval [ $\$ 5450, \$ 5500]$. The corresponding entry in our histogram is $(\$ 5450,82000 ; \$ 5500,71000)$. Each entry in the histogram consists of the co-ordinates of the two end points of a line segment.

(3). Since there may be too many intervals, requiring excessive storage, adjacent intervals are merged until a fixed number of, say $r$, intervals remain. The criterion to choose which adjacent intervals to merge is given by the Greedy Merge algorithm [13]. For every two adjacent intervals, the combined interval contains a straight line with a least square approximation to the tuples within it. The Greedy Merge algorithm chooses the combined interval with the smallest least square error. This process of combining adjacent intervals continues until $r$ intervals remain.

This method requires cooperation from local databases in the sense that it requires to have access to all data in each local database.

(B) MAXDIFF MHIST-2 (MHIST-2)

This method [19] operates as follows. Initially, there is a single region (in the case of two-dimensional data, this is a rectangle) containing all points. Then, a region (this is unique initially, but needs to be determined in later iterations of the algorithm) is chosen to be partitioned on one dimension into two regions such that the difference in density (the number of points within a region divided by the size of the region) between the two regions is the largest. (We also tried using frequency instead of density, but the result is worse.) This process is repeated until a fixed number of regions is reached. For each region in the his- 
togram, the number of points as well as two corner points defining the region are kept. This method also needs cooperation from local databases.

\section{(C) Frequent Queries (FQ)}

As pointed out in [2], queries usually follow a Zipfian distribution and histograms should be constructed utilizing the uneven query distribution. As a consequence, it is important to provide accurate estimation for frequently submitted queries. In [2], the queries are range-queries. Here, they are top-N queries.

We now propose a method to construct a special type of "histograms" which can be constructed without the cooperation of local database systems. In the Internet environment we cannot expect the needed histograms to be provided by the local e-commerce search engines. However, these engines will respond with results when a query is submitted. Thus, our approach is to submit user queries to the different databases (this has to be done anyway, so not much additional overhead is involved) and to construct the "histogram" based on the returned results of the frequently submitted queries. Our intuition is that if precise information is kept for data which are frequently accessed, then accuracy of estimation will be obtained. It is well-known that in realistic situations, data in a database are unevenly accessed with small amount of data heavily accessed but most other data lightly accessed. Since heavily accessed data are likely due to frequently submitted queries, we proceed to identify such queries. Ideally, we can keep track of all submitted queries and for each distinct submitted query, we record a frequency of submission. The queries can be sorted in descending order of frequency and the $\mathrm{k}$ most frequently submitted queries are retained to construct a histogram. This approach may not be practical, as the number of submitted queries is very large, which requires potentially unbounded storage. In order to approximate the ideal scheme with fixed amount of storage, we utilize two lists: Freq-Q and Infreq-Q. The Infreq-Q list serves as a filter. Only queries which have been submitted twice or more within a certain period of time can enter the Freq-Q list. We want to eliminate queries, each of which is submitted more than once but not too many times from the Freq-Q list. This is accomplished by the transposition scheme [20] in which every time an element is accessed, it is interchanged with the next element. It has been shown analytically $[20,15]$ that the transposition schema is a very good approximation to the ideal situation where records are arranged in descending order of frequencies of access. The transposition method is used to retain the frequently submitted queries. The same principle is used to retain the frequently accessed best matched tuples of the queries. Note that it is possible multiple distinct queries access the same data. We now describe our approach in more detail as follows.

Maintain a linked list of frequent-queries, Freq-Q, a linked list of best matched tuples, BMT, to some of the queries in Freq-Q and a queue of queries, each of which occurs only once within a period of time, Infreq-Q. The three data structures are all of limited fixed sizes. Initially, all three data structures are empty. When a query $Q$ arrives, if it is absent from both Infreq- $Q$ and Freq-Q, and Infreq-Q is not full, it is inserted into Infreq-Q. This indicates that the query is submitted exactly once. If Infreq-Q is full, then $\mathrm{Q}$ is inserted and the first (i.e., the oldest) query in Infreq-Q is removed.

If the query is found in Infreq-Q, then it is removed from Infreq-Q and inserted into Freq-Q. If Freq-Q is not at least $x \%$ full for some $x$ (say 90 ), then the query is inserted at the end of Freq-Q else it is inserted at the $x \%$ position towards the end (allowing some time for it to be moved forward, if it is accessed again. This causes the query at the end of Freq-Q to be dropped.). The top $N$ tuples of $\mathrm{Q}$ are found from the databases. From each database that contains at least one of these $N$ tuples, the best matched tuple for the database denoted by $B(Q, D)$ is retained. The set of $B(Q, D)$ 's from different databases together with the corresponding database IDs are placed into the linked list BMT. In addition, the distance of the N-th best matched tuple to $\mathrm{Q}, \mathrm{N}$-distance, is attached to the query $\mathrm{Q}$ in the linked list Freq-Q. If BMT is not at least $x \%$ full, then $\{\mathrm{B}(\mathrm{Q}, \mathrm{D})\}$ is inserted at its end else it is inserted at the $x \%$ position towards the end, possibly causing some end elements to be removed from the linked list.

If the query is found in Freq-Q, then it is moved up one position in the list (i.e., interchanged with the element in front of it). Each of the best matched tuples of $\mathrm{Q}$ in the linked list BMT, which can be determined using the distance of the $\mathrm{N}$-th best matched tuple of $\mathrm{Q}$ and with a data structure such as G-tree [14], is moved up one position in BMT. The actions in Freq-Q and BMT are to move frequently submitted queries and the frequently accessed tuples towards the fronts of the two lists. The process of processing queries in Infreq-Q, Freq-Q and BMT continues until there are no substantial changes in the set of queries in the front part of Freq-Q. In other words, if the frequent queries submitted by the users become stable, the construction of the "histogram" (which is the linked list of best matched tuples, BMT, together with the frequent queries in Freq-Q) can stop. If user access pattern needs to be monitored for changes, the construction process continues.

When a query $\mathrm{Q}$ is submitted to be processed, it is checked against the list Freq-Q. If it is there, $\{B(Q, D)\}$ (the best matched tuple from each desired database) in BMT are retrieved and the database IDs are used to decide which databases should be accessed to process the query. (It is possible that some elements in $\{\mathrm{B}(\mathrm{Q}, \mathrm{D})\}$ are removed from BMT or some of the best matched tuples in various databases have been modified. That situation can be detected by processing the 
query and by comparing the distance of the N-th retrieved best matched tuple against $\mathrm{N}$-distance. If they are different, additional databases need to be searched to obtain the actual top $N$ tuples. The new B(Q,D)'s need to be inserted into the linked list BMT and the $\mathrm{N}$-distance in Freq-Q needs to be updated.) If the top $N$ tuples of the query are unchanged and all elements in the set $\{B(Q, D)\}$ are present in BMT, then all top $N$ tuples of $\mathrm{Q}$ will be retrieved.

If a query is not found in Freq-Q, the $N$ best matched tuples from BMT are obtained. They are sorted in ascending order of distance and their corresponding database IDs are used in the merge algorithm (see Section 3.4) to retrieve the top $N$ tuples from the databases. Here, there is no guarantee that all of the top $N$ tuples of the query will be retrieved.

(D) Independent Linear Approximation (ILA) In this approach, there is a histogram for each attribute and the values under different attributes are assumed to be independent. This is the approach used in [23]. Again, cooperation of local systems is needed to construct the histograms.

\subsection{Criterion for Ranking Databases Opti- mally}

We now sketch how databases containing the top $N$ tuples should be selected. First, all databases should be ranked with respect to the query (Proposition 1). Next, the proper databases (and the proper tuples) are selected using the merge algorithms to be described in Section 3.4,

Definition 1 Suppose a user is interested in retrieving the $N$ best matched tuples to a submitted query $Q$. Databases $\left\{D_{i}, 1 \leq i \leq n\right\}$ are optimally ranked in the order $D_{1}, D_{2}, \ldots, D_{n}$, if for every $N$, there exists at such that $D_{1}, D_{2}, \ldots, D_{t}$ collectively contain all the $N$ best matched tuples of $Q$ and each $D_{i}, 1 \leq i \leq t$, contains at least one of the $N$ best matched tuples.

The following proposition provides a criterion for ranking databases optimally [22]. (In [22], the result is stated in terms of similarity. Since similarity and distance are inverses of each other, the databases are arranged in descending order of similarities in [22].)

Proposition 1 For a given query $Q$, if databases are ranked in ascending order of the distances of the best matched tuples to $Q$, then they are ranked optimally with respect to $Q$.

Example 2 Suppose there are 4 databases $D_{1}, D_{2}, D_{3}$ and $D_{4}$. Suppose that the distances of the best matched tuples in these databases to query $Q$ are $0.8,0.6,0.3$, and 0.5 , respectively. Then, for query $Q$, the databases should be ranked in the order $D_{3}, D_{4}, D_{2}, D_{1}$.

This proposition cannot be used as is because it is impractical to actually search each database in order to find the distance of its best matched tuple for each query. Therefore, the distance will need to be estimated as described in the next subsection.

\subsection{Estimate Smallest Distance to Query}

The estimation of the distance of the best matched tuple in each database is carried out based on the histogram(s) of the database.

First, consider the case when the histogram is constructed using the linear approximation (LA) approach. Consider the query (price $=p$, mileage $=q$ ) From the histogram of a database, find the interval $I$, which contains price $=p$. From the interval, the distance of the query point to the closest point in the straight line is computed. Details can be found in [24].

The closest point to the query point may or may not appear in the interval, $I$. To improve the accuracy, a fixed number of intervals, each of which is either $I$ or close to $I$ are chosen. Then the distance of the closest point is the minimum of the distances computed in the various intervals. Since a fixed number of intervals is used, then only constant time is needed. However, finding the interval containing the query point takes $O(\log |F|)$ times. In summary, for a two-attribute relation, the estimation time is $O(\log |F|)$.

The above scheme for histogram construction and estimation can be generalized to n-dimensions, $n \geq 2$. Intervals and straight lines in 2 dimensions are replaced by hyper rectangles and hyper planes in higher dimensions.

Consider other types of histograms. For MHIST-2, to estimate the distance of the best matched tuple to a query, it is assumed that the points within a region are uniformly distributed. The idea of selecting databases for a query using the FQ method is sketched in Section 3.1 (see the last two paragraphs of item (C)). For ILA, the method proposed in [23] to perform the estimate can be used. This method has an exponential complexity.

\subsection{Algorithms to Select Tuples from Ranked Databases}

In this section, two algorithms for selecting tuples from the ranked databases are given. The first algorithm, Merge-1, was proposed in [23] and is included for comparison purpose; The second algorithm is MIN-2. It will be slightly modified to become MOD-MIN-2. It will be shown that Merge- 1 is more efficient (i.e., fewer databases are likely to be accessed for a given query), while the MOD-MIN-2 is more effective (i.e., more actual top $N$ tuples are likely to be obtained).

\subsubsection{Algorithm Merge-1}

Let databases be ranked in the order $\left[D_{1}, D_{2}, \ldots, D_{n}\right]$. Let $N$ be the number of tuples the user desires. The databases are accessed in the order in which they are ranked, one at a time. (In practice, the first few 
highest ranked databases may be accessed in parallel.) Suppose the first $t$ databases have been accessed and $d$ is the maximum value of the distances of the best matched tuples, one from each of these $t$ databases. Tuples from these $t$ databases with distance $\leq d$ are retrieved. If $N$ or more tuples have been retrieved, then they are sorted in ascending order of distances, the first $N$ tuples are returned to the user and the process is terminated; else the next database is accessed, $d$ is updated and tuples from these $(t+1)$ databases with distance $d$ are retrieved. Two remarks about the above merge algorithm:

1. Whenever a database is accessed for the first time, its top $N$ tuples are retrieved and cached at the global site. All subsequent "interactions" between the accessed database and the global site will actually take place at the global site only. This ensures that each database is accessed at most once.

2. In the future, relational databases for ecommerce will support top $N$ queries of different types as discussed in Section 2. Thus, in this paper, we assume that local distance functions are identical to the corresponding global distance functions. If this is not the case, each invoked database is required to return $N$ or slightly more local top $N$ tuples. These tuples are then re-ranked using the global distance function.

The remarks apply to all the merge algorithms.

\subsubsection{Algorithm MIN-2}

The Merge-1 algorithm retrieves at least one tuple from each database it accesses, because when database $D_{i}$ is accessed, tuples with distances less than or equal to the maximum of the distances of the best matched tuples in the accessed databases are retrieved. If an accessed database does not contain any of the $\mathrm{N}$ best matched tuples of the query, some of its tuples will still be retrieved. To remedy this situation, the MIN-2 algorithm allows such a bad database to be skipped over, as long as each of its two adjacent databases contains at least one of the $N$ best matched tuples.

As before let these databases be ordered in $D_{1}, D_{2}, \ldots, D_{n}$. MIN-2 operates as follows. Access the top two ranked databases $D_{1}$ and $D_{2}$ to obtain the best matched tuple from each of them. Let the distances of the best matched tuples be denoted by $d_{1}$ and $d_{2}$. Let the minimum of the two values be threshold $d=\min \left\{d_{1}, d_{2}\right\}$. Tuples from these two databases with distances $\leq d$ are retrieved. If $N$ or more tuples are retrieved, sort them in ascending order of the actual distances; output the first $N$ tuples and terminate; else, access the next database, $D_{i}$ and let the actual distance of the best matched tuple in this database be $d_{i}$. Re-compute $d=\min \left\{d_{i}, d_{i-1}\right\}$, where $d_{i-1}$ is the distance of the best matched tuple in the last accessed database. Retrieve from all accessed databases tuples with distance $\leq d$. (Tuples which have been retrieved previously will not be retrieved again.) If $N$ or more tuples have been retrieved, sort them in ascending order of the actual distances; output the first $N$ tuples and terminate; else, the process is repeated until $N$ or more tuples are retrieved. Then output the $N$ tuples with the smallest distances.

Proposition 2 Let the distance of the $N$-th best matched tuple, $N_{d}$, be distinct. If the first $t$ databases collectively contain $T$, the set of the top $N$ tuples and each of these databases contains at least one tuple in $T$, then algorithm $M I N-2$ will retrieve all tuples in $T$ by accessing at least $t+1$ databases and at most $t+2$ databases.

An example in [24] shows that the condition "the distance of the $N$-th best matched tuple is distinct" is necessary for guaranteeing the retrieval of all the top $N$ tuples. All proofs in this section can be found in [24].

Although in general the MIN-2 algorithm has higher retrieval effectiveness than the Merge- 1 algorithm, the following example shows that it is possible that MIN-2 has a lower effectiveness in some situation.

Example 3 Suppose site 1 has a single tuple with distance 0.6 , site 2 has a single tuple with distance 0.55 , site 3 has a tuple with distance 0.52 , site 4 has 3 tuples with distances $0.4,0.45$ and 0.5 and site 5 has a single tuple with distance 0.3. Let the number of tuples to be retrieved be 4 . The original algorithm, Merge-1, after visiting site 4, retrieves the tuples with distances $0.6,0.55,0.52,0.4,0.45$, and 0.5 . It then returns the 4 tuples with distances $0.4,0.45,0.5$ and 0.52 . After visiting site $4, \mathrm{MIN}-2$ retrieves the tuples with distances $0.55,0.52$ and 0.4 only. Therefore, it needs to proceed further. After visiting site 5 , it retrieves the tuples with distances $0.55,0.52,0.4$ and then 0.3. Clearly, the 4 best matched tuples are those with distances $0.3,0.4,0.45$ and 0.5 . The original algorithm, Merge-1, retrieves 3 of them, while the MIN-2 algorithm retrieves only 2 of them.

One possible way for a remedy is as follows. We proceed exactly the same as MIN-2. At the same time, we also consider the set of tuples, $\mathrm{P}$, which is retrievable by the original algorithm, Merge-1. At any site, the number of tuples retrieved by the modified MIN-2 algorithm is identical to that of MIN-2. However, if there are tuples in $\mathrm{P}$ which have lower distances than those of tuples retrievable by MIN-2, replace the latter tuples by the former tuples.

Example 4 Continue on the last Example. After visiting the first 4 sites, the new algorithm retrieves the tuples with distances $0.55,0.52$ and 0.4 . But, it also considers the set of tuples retrievable by Merge- 1 . This includes the set of tuples with distances 0.45 and 0.5 . Since the tuples having distances 0.55 and 0.52 are not as good as tuples with distances 0.45 and 0.5 , they are replaced by the latter two tuples. Thus, after visiting 4 
sites, the set of tuples retrieved by the modified MIN2 have distances $0.4,0.45$ and 0.5 . After visiting site 5 , we get the four tuples with distances $0.3,0.4,0.45$ and 0.5. This set of tuples has higher retrieval effectiveness than the set of tuples retrieved by the original algorithm, Merge-1.

The modified algorithm, MOD-MIN-2, initially retrieves the same set of documents as MIN-2, except that documents with larger distances are replaced by documents with smaller distances in Merge-1. Both MOD-MIN-2 and MIN-2 retrieve the same number of documents. See [24] for details.

Proposition 3 The new algorithm, MOD-MIN-2, has effectiveness at least as high as that of algorithm Merge-1, when the number of databases is at least $2 N$.

\section{Experiments}

The data and query collections used in the experiments are described in Section 4.1. In Section 4.2, two measures of retrieval, one reflecting the effectiveness and the other reflecting the efficiency, are provided. Experimental results are provided in Section 4.3.

\subsection{Data Sets and Query Sets}

Two data sets are used.

(1). Used car data were collected from Excite's Classification 2000 website with the following conditions: Make = "any", Model = "all models", Year = "1900 to $2000 "$, Price $=" \$ 500$ to $\$ 27,000 "$. There are 32,449 tuples. The tuples are arbitrarily assigned to 28 databases without duplication. Two types of queries are used and they are 2-D queries and 3-D queries. 2-D queries are two-attribute queries involving price and mileage and 3-D queries involve price, mileage and age. The values associated with the two attributes are chosen to reflect reality and some diversification. Ideally, if the mileage is high, the price must be low; if the mileage is low, the price must be high. A set of 50 queries is generated on price and mileage. This query set is used in all the experimental runs. Each query is interpreted as a standard top- $N$ query, a generalized top- $N$ query and a two sided-generalized top- $N$ query. For each interpretation, the two "distance functions", namely, the Euclidean distance and the Manhattan distance functions are used.

(2). Forest Cover data were obtained from UC Irvine (ftp://ftp.ics.uci.edu/pub/machine-learningdatabases/covtype/). There are 581,012 tuples which are randomly assigned to 50 databases. Three types of queries are utilized. 2-D queries involve the attributes elevation and slope; $3-\mathrm{D}$ queries use an additional attribute horizontal_distance_to_road; 5-D queries use two more attributes horizontal_distance_to_hydro and horizontal_distance_to_fire_points.

A set of 10,000 distinct queries that satisfy the Zipfian distribution are randomly generated, i.e., the $i$ - th most frequently submitted query has probability $\frac{1 / i}{\sum_{j=1}^{K} 1 / j}$, where $K=10,000$ is the number of distinct queries. This is used for the FQ method. For all other methods, the 50 queries having the highest frequencies of submission are used. (If these 50 queries were used by the FQ method, then $100 \%$ accuracy would be achieved.) Each method is assigned the same amount of space for histogram construction. It is about $2 \%$ of the size of the data for the forest-cover data set and also about $2 \%$ of the size of the data for the used-car data set.

\subsection{Performance Measures}

Performances are measured by the effectiveness and the efficiency of retrieval. The former is measured by the number of the retrieved tuples that are among the actual $N$ best matched tuples divided by $N$. If the quantity is $100 \%$, then all of the $N$ best matched tuples are retrieved. Higher percentages indicate higher retrieval effectiveness. Efficiency is measured by two factors: the first factor is the ratio of the number of databases accessed to the actual number of databases containing the $N$ best matched tuples. If the ratio is $100 \%$, then the number of databases accessed is the same as the number of databases containing the $N$ best matched tuples although not necessarily the same set of databases is accessed. A ratio exceeding $100 \%$ indicates inefficiency. Lower ratios indicate higher efficiency. The second factor is the time to determine the ordering of the databases with respect to the query by executing against the histogram. (This is an overhead versus sending a query to all databases.) Since the FQ method seems to be the only viable method (see the next subsection), we shall report that factor for the FQ Method only. Ideal retrieval is achieved when accuracy is $100 \%$, efficiency is $100 \%$ and the time to order the databases for each query is small.

\subsection{Experimental Results}

In Section 4.3.1, we use the used car data set to show that the new merge method, MOD-MIN-2, used in conjunction with Linear Approximation Method (LA) is much better than the previous merge method used in conjunction with the Independent Linear Approximation Method (ILA). (Experimental results showing that the new merge method by itself yields substantial improvement over the previous merge method are obtained, but they are not shown here for lack of space.) Thus, we eliminate the ILA method from further consideration. In Section 4.3.2, the three methods LA, MHIST-2 and FQ are compared with respect to the used-car data set and the forest-cover data set. It will be shown that in most situations, the FQ method achieves significantly higher accuracy than the other two methods for both 2-D and 3-D queries and has high accuracy in all situations. 5-D queries using the 
Method LA Method ILA

\begin{tabular}{|c|c|c|c|c|c|}
\hline $\mathrm{N}$ & Accu. & Effic. & Accu. & Effic. & \#Q \\
\hline 5 & 70.4 & 153.7 & 28.4 & 75.6 & 50 \\
10 & 74.2 & 135.2 & 31.6 & 68.9 & 50 \\
20 & 83.6 & 118.9 & 37.3 & 59.9 & 50 \\
30 & 86.7 & 116.5 & 42.3 & 60.3 & 50 \\
\hline
\end{tabular}

Table 1: Standard Manhattan Distance

\begin{tabular}{|c|c|c|c|c|c|}
\multicolumn{5}{c}{ Method LA } & \multicolumn{3}{c|}{ Method ILA } \\
\hline $\mathrm{N}$ & Accu. & Effic. & Accu. & Effic. & \#Q \\
\hline 5 & 69.6 & 156.9 & 27.6 & 73.8 & 50 \\
10 & 77.6 & 137.6 & 30.4 & 67.1 & 50 \\
20 & 86.5 & 124.4 & 36.5 & 58.3 & 50 \\
30 & 88.8 & 119.6 & 39.9 & 60.9 & 50 \\
\hline
\end{tabular}

Table 2: Standard Euclidean Distance

forest-cover data set are executed by the FQ method and superior results are also obtained. (The used car data set does not have 5 attributes requiring approximation. Attributes such as the model and the make of a car require exact satisfaction, not approximate satisfaction.) In the above experiments, a histogram is constructed for each combination of distance function and query dimension. In Section 4.3.3, we also experiment with the situation that a single histogram is used for all types of queries (having different dimensions and/or having different distance functions). The FQ-method continues to do well in this situation. Finally, the original algorithm and the new merge algorithm, MOD-MIN-2, are used for different query types to yield both high accuracy and high efficiency. The average time to order the databases for each query for the FQ method is reported to be reasonable.

\subsubsection{Comparing ILA with LA Using the Used Car Data Set}

A previous solution [23] consists of the Merge- 1 algorithm and a method (with an exponential time complexity) to estimate the distance of the best matched tuple based on the ILA histograms. The LA method consists of the new merge algorithm, MOD-MIN-2, the LA histograms and a new algorithm (with low polynomial time complexity [24]) for estimation.

Tables 1 and 2 give the accuracies and the efficiencies of the two methods for 2-D queries when the standard Manhattan and the standard Euclidean distance functions are employed. (In all tables, \#Q stands for "number of queries used".) It clearly demonstrates that the LA method is much better than the ILA method. Furthermore, the ILA has too low an accuracy to be useful in practice. (In [23], much higher accuracy was reported for the ILA method. However, much more space was allocated for histogram construction in [23] than in here.)

Tables 3 and 4 show the corresponding results for
Method LA Method ILA

\begin{tabular}{|c|c|c|c|c|c|}
\hline $\mathrm{N}$ & Accu. & Effic. & Accu. & Effic. & \#Q \\
\hline 5 & 100 & 193.5 & 42.0 & 147.0 & 50 \\
10 & 100 & 169.2 & 48.8 & 181.0 & 50 \\
20 & 100 & 153.0 & 49.7 & 189.0 & 50 \\
30 & 99.3 & 141.8 & 52.9 & 158.5 & 50 \\
\hline
\end{tabular}

Table 3: Generalized Manhattan Distance

Method LA Method ILA

\begin{tabular}{|c|c|c|c|c|c|}
\hline $\mathrm{N}$ & Accu. & Effic. & Accu. & Effic. & \#Q \\
\hline 5 & 83.6 & 141.7 & 49.6 & 98.5 & 50 \\
10 & 83.4 & 124.2 & 54.6 & 84.8 & 50 \\
20 & 86.7 & 113.2 & 57.1 & 76.7 & 50 \\
30 & 89.6 & 110.1 & 60.2 & 76.2 & 50 \\
\hline
\end{tabular}

Table 4: Two-sided Manhattan Distance

the methods LA and ILA for the Generalized Manhattan Distance function and the Two-sided Manhattan Distance. When an estimation method, a histogram construction method and a merge algorithm are fixed, the differences in accuracy between the Manhattan distance and the Euclidean Distance functions are usually 1 to 2 percentages. As a consequence, we do not show the corresponding results for the Generalized Euclidean Distance function and the Two-sided Euclidean Distance function. Again, for all these distance functions, the LA method with the new merge algorithm has much higher accuracy than the ILA method with the original merge algorithm. Based on the results identified in Tables 1-4, it is clear that significantly higher accuracies can be achieved by having a histogram construction process which incorporates the dependencies between attributes and utilizing the new merge algorithm.

\subsubsection{Comparing LA, MHIST-2 and FQ}

In the second set of experiments, the following issues are addressed: (1) Are there significant differences in accuracies among LA, MHIST-2 and FQ? (2) How do these methods perform when the number of dimensions increases? It is known that maintaining accuracies in high dimensional space is very challenging. For both issues, both the used-car data set and the forestcover data set are used. For issue 1, only 2-D queries are submitted and answered. For issue 2, the number of dimensions is increased to 3 and then to 5 .

From Tables 1-5 involving the used-car data set, the best performance in accuracy is achieved by the FQ method; it is followed by the LA method and then the MHIST-2 method. The differences in accuracy between the FQ method and the LA method are very significant for the Standard Manhattan distance and the Standard Euclidean distance functions, ranging from $20 \%$ for 5 best matched tuples to about $6-9 \%$ for 30 best matched tuples. The high accuracy of the FQ 
Method MHIST-2 Method FQ

\begin{tabular}{|c|c|c|c|c|c|c|}
\hline $\mathrm{N}$ & Accu. & Effic. & \#Q & Accu. & Effic. & \#Q \\
\hline 5 & 48.0 & 162.5 & 50 & 90.9 & 139.2 & 10000 \\
10 & 58.6 & 137.5 & 50 & 93.3 & 136.3 & 10000 \\
20 & 72.7 & 122.9 & 50 & 95.0 & 122.4 & 10000 \\
30 & 81.3 & 123.3 & 50 & 95.5 & 118.9 & 10000 \\
\hline
\end{tabular}

Table 5: Standard Manhattan Distance, 2-D Used-Car Data Set

Method MHIST-2

\begin{tabular}{|c|c|c|c|c|c|c|}
\hline $\mathrm{N}$ & Accu. & Effic. & \#Q & Accu. & Effic. & \#Q \\
\hline 5 & 49.2 & 154.0 & 50 & 89.3 & 139.7 & 10000 \\
10 & 56.6 & 134.8 & 50 & 91.8 & 137.6 & 10000 \\
20 & 69.4 & 123.0 & 50 & 93.6 & 120.5 & 10000 \\
30 & 76.1 & 121.0 & 50 & 94.2 & 121.9 & 10000 \\
\hline
\end{tabular}

Table 6: Standard Euclidean Distance, 2-D Used-Car Data Set

method for the Standard Manhattan and the Standard Euclidean distance functions is also confirmed when the Forest Cover data set is used, as seen in Tables 9-10. While the MHIST-2 method is inferior to the LA method for the used-car data, it is better than the latter method for the forest cover data set (see Tables 9-11). For both data sets, the FQ method is much better than the other methods for the two standard distance functions. For both data sets, the same is true in efficiency.

For the Generalized Manhattan distance function, the 3 methods LA, MHIST-2 and FQ all averaged higher than $95 \%$ accuracy (see Tables 3 and 7 ). These methods also perform similarly for the generalized Euclidean distance function (the results are not shown here due to space limitation). Thus, all these methods perform extremely well for these two generalized distance functions, although the FQ method performs slightly worse. In terms of efficiency, the three methods are comparable for the used-car data set. For each of the Two-Sided distance functions, each method performs better than its corresponding Standard distance function but worse than the corresponding Generalized distance function (See for example, the results of FQ in Tables 5, 7 and 8). For these Two-sided distance functions, the FQ method has superior performance (see Table 8. Results for the FQ method involving the Two-sided Euclidean distance function are similar

\begin{tabular}{|c|c|c|c|c|c|c|}
\multicolumn{4}{c}{ Method MHIST-2 } & \multicolumn{3}{c|}{ Method FQ } \\
\hline $\mathrm{N}$ & Accu. & Effic. & \#Q & Accu. & Effic. & \#Q \\
\hline 5 & 97.2 & 200.6 & 50 & 100 & 196.1 & 10000 \\
10 & 96.2 & 172.5 & 50 & 93.8 & 163.9 & 10000 \\
20 & 94.7 & 156.5 & 50 & 98.2 & 153.4 & 10000 \\
30 & 98.7 & 159.0 & 50 & 99.0 & 144.1 & 10000 \\
\hline
\end{tabular}

Table 7: Generalized Manhattan Distance, 2-D UsedCar Data Set

\begin{tabular}{|c|c|c|c|c|c|c|}
\multicolumn{4}{c}{ Method MHIST-2 } & \multicolumn{3}{c|}{ Method FQ } \\
\hline N & Accu. & Effic. & \#Q & Accu. & Effic. & \#Q \\
\hline 5 & 65.2 & 159.3 & 50 & 96.1 & 141.3 & 10000 \\
10 & 71.6 & 129.8 & 50 & 96.0 & 134.1 & 10000 \\
20 & 74.8 & 118.5 & 50 & 95.4 & 117.2 & 10000 \\
30 & 77.0 & 116.4 & 50 & 94.4 & 120.7 & 10000 \\
\hline
\end{tabular}

Table 8: Two-Sided Manhattan Distance, 2-D UsedCar Data Set

Method MHIST-2 Method FQ

\begin{tabular}{|c|c|c|c|c|c|c|}
\hline $\mathrm{N}$ & Accu. & Effic. & \#Q & Accu. & Effic. & \#Q \\
\hline 5 & 62.8 & 245.8 & 50 & 95.8 & 167.5 & 10000 \\
10 & 67.0 & 195.0 & 50 & 96.8 & 152.9 & 10000 \\
20 & 70.4 & 169.4 & 50 & 97.4 & 140.9 & 10000 \\
30 & 78.5 & 160.0 & 50 & 97.8 & 131.0 & 10000 \\
\hline
\end{tabular}

Table 9: Standard Manhattan Distance, 2-D Forest Cover Data Set

and are not shown.). In summary, the FQ method has excellent accuracies for both data sets and for all 6 distance functions.

We now report the results for the 3 methods involving 3 attributes using the Standard Manhattan Distance and the Standard Euclidean Distance only. The accuracy results for the Two-Sided distance function and the Generalized distance function will be higher, as observed earlier. We first report the results of the three methods on the forest-cover data set. As can be seen in Tables 12-13, the accuracies of the two methods LA and the MHIST-2 are too low to be acceptable, while the FQ method has accuracies above $95 \%$. Only the efficiency of the last method is reported. It is reasonably efficient. The results of the FQ method for the used-car data set are reported in Table 14. Although the results are worse than those reported for the same method with 2-D attributes, the accuracies remain high, from $83 \%$ to $91 \%$.

The results for the FQ method involving 5-D attributes are reported in Table 15 . (For the used-car data set, there are no 5-D top-N queries, as complete satisfaction is required for attributes such as model and make for cars.) In comparing the results in Table 15 to those in Tables 12-13, it is clear that there is essentially no difference in accuracy between 5-D attributes and 3-D attributes, although there is a deterioration in retrieval efficiency. Accuracies stay above

\begin{tabular}{|c|c|c|c|c|c|c|}
\multicolumn{4}{c}{ Method MHIST-2 } & \multicolumn{3}{c|}{ Method FQ } \\
\hline N & Accu. & Effic. & \#Q & Accu. & Effic. & \#Q \\
\hline 5 & 61.6 & 241.3 & 50 & 95.8 & 170.2 & 10000 \\
10 & 64.8 & 208.4 & 50 & 97.0 & 157.7 & 10000 \\
20 & 71.1 & 177.4 & 50 & 97.5 & 141.9 & 10000 \\
30 & 79.5 & 163.7 & 50 & 97.8 & 132.8 & 10000 \\
\hline
\end{tabular}

Table 10: Standard Euclidean Distance, 2-D Forest Cover Data Set 
Standard Euclidean Standard Manhattan

\begin{tabular}{|c|c|c|c|c|c|c|}
\hline $\mathrm{N}$ & Accu. & Effic. & \#Q & Accu. & Effic. & \#Q \\
\hline 5 & 64.4 & 197.3 & 50 & 66.4 & 198.0 & 50 \\
10 & 67.4 & 158.2 & 50 & 67.0 & 154.0 & 50 \\
20 & 67.3 & 134.7 & 50 & 67.6 & 133.3 & 50 \\
30 & 69.7 & 120.0 & 50 & 67.9 & 114.4 & 50 \\
\hline
\end{tabular}

Table 11: Method LA, 2-D Forest Cover Data Set

\begin{tabular}{l|l|l} 
Method LA & Method MHIST-2 & Method FQ
\end{tabular}

\begin{tabular}{|c|c|c|c|c|}
\hline $\mathrm{N}$ & Accu. & Accu. & Accu. & Effic. \\
\hline 5 & 34.0 & 46.0 & 95.8 & 170.2 \\
10 & 36.8 & 50.8 & 97.0 & 157.7 \\
20 & 43.1 & 51.9 & 97.5 & 141.9 \\
30 & 45.2 & 57.1 & 97.8 & 132.8 \\
\hline
\end{tabular}

Table 12: Standard Euclidean Distance, 3-D Forest Cover Data Set

$95 \%$ on the average. Based on the results reported here, the FQ method is the only method that produces high accuracy for 2-D, 3-D and 5-D attributes for both data sets. Its efficiency is also very reasonable.

\subsubsection{A single histogram for a mixture of queries}

In reality, users submit different types of queries (different dimensions, different distance functions) at different times. Our last experiment involves 2-D, 3-D and 5-D queries with the 6 different distance functions. Each of the 2-D, 3-D and 5-D type of queries has approximately the same number of occurrences; each of the 6 distance functions involved in the queries also has approximately the same number of occurrences. 10,000 such distinct queries satisfying the Zipfian distribution are generated. 20,000 such queries, generating from the same distribution are used to construct the histogram using no more than $2 \%$ of the space of the data. Then, 1,000 queries are submitted to test the accuracy and the efficiency of the FQ method. As indicated in the left part of Table 16, accuracy ranges from $95.7 \%$ to $97.7 \%$. However, efficiency ranges from $205 \%$ to $146 \%$. After examining the accuracies of the queries, it is found that for the Standard Euclidean Distance queries and the Standard Manhattan Distance queries, the use of the new merge algorithm gives approximately $3-4 \%$ improvement in accuracy over the

\begin{tabular}{l|l|l} 
Method LA & Method MHIST-2 & Method FQ
\end{tabular}

\begin{tabular}{|c|c|c|c|c|}
\hline $\mathrm{N}$ & Accu. & Accu. & Accu. & Effic. \\
\hline 5 & 45.6 & 41.6 & 95.8 & 167.5 \\
10 & 49.0 & 45.0 & 96.8 & 152.9 \\
20 & 57.0 & 50.8 & 97.5 & 140.7 \\
30 & 60.9 & 54.9 & 97.8 & 131.0 \\
\hline
\end{tabular}

Table 13: Standard Manhattan Distance, 3-D Forest Cover Data Set
Standard Manhattan Standard Euclidean

\begin{tabular}{|c|c|c|c|c|}
\hline $\mathrm{N}$ & Accu. & Accu. & Accu. & Effic. \\
\hline 5 & 83.0 & 144.7 & 83.4 & 144.7 \\
10 & 86.7 & 127.9 & 87.2 & 128.5 \\
20 & 90.5 & 120.6 & 90.1 & 118.4 \\
30 & 92.6 & 118.7 & 91.7 & 118.6 \\
\hline
\end{tabular}

Table 14: Method FQ, 3-D Used-Car Data Set

original merge algorithm, while for all other queries, there is negligible difference in accuracy between the merge algorithms. Thus, the experiment is repeated in which the new merge algorithm is used for the former two types of queries and the original merge algorithm is used for the remaining four types of queries. The right part of Table 16 shows that such a strategy yields essentially the same accuracy, while efficiency improves significantly. The average time to determine the ordering of the databases for a query using the FQ method is 37.8 msec. Thus, the FQ method can order the databases accurately in reasonable time.

\section{Conclusion}

In this paper, we presented a two-step solution for finding the $N$ best matched tuples for a given query in a distributed relational database environment. Four different methods for implementing the first step, i.e. ranking databases based on the estimated distance of the best matched tuple in each database, were compared. In addition, the new merge algorithm (MODMIN-2) was proposed for the second step. This algorithm was shown to achieve higher effectiveness than the merge algorithm (Merge-1) proposed in [23]. Our experimental results show that the $\mathrm{FQ}$ method when used in conjunction with the new merge algorithm achieves high accuracies in all cases. Furthermore, the method does not require cooperation from various databases, in contrast to all other histogram construction methods [21]. Thus, the approach suggested here is very promising.

We believe that the proposed solution will be useful in large scale distributed systems (e.g., the Internet) where the same or similar goods and services are offered by numerous vendors and the users need to seek the best results among these offerings without exerting excessive burden on him/her and on the computing and communication resources. An example application can be the long term care insurance where there are about 120 companies selling such insurance and the specification of the desired care required by an individual/couple can be complicated (i.e., require top- $\mathrm{N}$ query processing).

Acknowledgment: This work is supported in part by the following grants: IIS-9902872, IIS-9902792, EIA-9911099, IIS-0208574, IIS-0208434 and ARO-25-30267. We are also grateful to Wensheng Jia who implemented some of the programs. 
Standard Manhattan Standard Euclidean

\begin{tabular}{|c|c|c|c|c|}
\hline $\mathrm{N}$ & Accu. & Accu. & Accu. & Effic. \\
\hline 5 & 95.2 & 214.1 & 94.4 & 233.4 \\
10 & 96.2 & 182.0 & 95.4 & 202.7 \\
20 & 97.1 & 162.7 & 96.7 & 174.1 \\
30 & 97.2 & 152.5 & 96.6 & 161.0 \\
\hline
\end{tabular}

Table 15: Method FQ, 5-D Forest Cover Data Set

\begin{tabular}{|c|c|c|c|c|}
\multicolumn{3}{c}{ MOD-MIN-2 } & \multicolumn{1}{c|}{ Merge-1 \& MOD-MIN-2 Combined } \\
\hline N & Accu. & Effic. & Accu. & Effic. \\
\hline 5 & 95.7 & 205.1 & 95.7 & 153.0 \\
10 & 96.9 & 169.7 & 96.7 & 133.8 \\
20 & 97.5 & 156.0 & 97.4 & 126.3 \\
30 & 97.7 & 146.4 & 97.6 & 120.1 \\
\hline
\end{tabular}

Table 16: Method FQ

\section{References}

[1] N. Bruno, S. Chaudhuri and L. Gravano. Performance of Multiattribute Top- $k$ queries on Relational Systems. Technical report, Columbia University, Computer Science Dept, 2000.

[2] N. Bruno, S. Chaudhuri and L. Gravano. STHoles: A Multidimensional Workload-Aware Histogram. ACM SIGMOD Conference, 2001.

[3] M. Carey and D. Kossmann. Reducing the Braking Distance of an SQL Query Engine. VLDB Conference, 1998.

[4] S. Chaudhuri and L. Gravano. Evaluating Top- $k$ Selection queries. VLDB Conference, 1999.

[5] A. Deshpande, M. Garofalakis and R. Rastogi. Independence is good: Dependency-Based Histogram Synopses for High Dimensional Data. SIGMOD Conference, 2001.

[6] D. Donjerkovic and R. Ramakrishnan. Probabilistic Optimization of Top $N$ Queries, VLDB Conference, 1999.

[7] A. Fu, P. Chan, Cheung Y and Moon, Y. Dynamic $V P$ tree indexing for $N$-nearest neighbor search given pairwise distances, VLDB Journal, 2000.

[8] L. Gravano, and Garcia-Molina, H. Merging ranks from heterogeneous internet sources. VLDB Conference, 1997.

[9] D. Gunopulos, G. Kollios, V.J. Tsotras and C. Domeniconi. Approximating multi-dimensional aggregate range queries over real attributes. ACM SIGMOD Conference, 2000.

[10] V. Hristidis, N. Koudas, Y. Papakonstantinou. PREFER: A System for the Efficient Execution of Multi-parametric Ranked Queries. ACM SIGMOD Conference, 2001.
[11] Y. Ioannidis and V. Poosala. Histogram-based Approximation of Set-valued Query Answers. VLDB Conference, 1999.

[12] W. Kiessling, G. Koestler. Preference SQL - Design, Implementation, Experiences. VLDB Conference, 2002.

[13] A. Konig and G. Weikum. Combining Histograms and Parametric Curve Fitting for FeedbackDriven Query Result-Size Estimation. VLDB Conference, 1999.

[14] A. Kumar. G-Tree: A New Data Structure for Organizing Multidimensional Data. IEEE TKDE, 6(2), April 1994.

[15] K. Lam, M. Siu, and C. Yu. A Generalized Counter Scheme. J. of Theoretical Computer Science, Sept. 1981, pp. 271-278.

[16] A. Levy, A. Rajaraman, and J. Ordille. Querying Heterogeneous Information Sources Using Source Descriptions. VLDB Conference, 1996.

[17] L. Liu. Query Routing in Large-scale Digital Library Systems. 15th International Conference on Data Engineering (ICDE'99), March 1999.

[18] M. Muralikrishna and D. J. DeWitt. Equi-Depth Histograms For Estimating Selectivity Factors For Multi-Dimensional Queries. ACM SIGMOD Conference, 1988.

[19] V. Poosala and Y. Ioannidis. Selectivity Estimation Without the Attribute Value Independence assumption. VLDB Conference, 1997.

[20] R. Rivest. On Self-Organizing Sequential Search Heuristics. CACM 19(2): 63-67, 1976.

[21] N. Thaper, S. Guha, P. Indyk, and N. Koudas. Dynamic Multidimensional Histogram. ACM SIGMOD Conference, 2002.

[22] C. Yu, W. Meng, W. Wu, K. Liu. Efficient and Effective Metasearch for Text Databases Incorporating Linkages among Documents. ACM SIGMOD Conference, May 2001.

[23] C. Yu, P. Sharma, W. Meng and Y. Qin. Databases Selection for Processing $k$ Nearest Neighbors Queries in Distributed Environments. 1st ACM/IEEE-CS joint conf. on DL, 2001.

[24] C. Yu, G. Philip and W. Meng Distributed Top-N Query Processing with Possibly Uncooperative Local Systems, Technical Report, Dept. of CS, University of Illinois at Chicago, 2003 (available at http://www.cs.binghamton.edu/ meng/pub.d/ vldb03long.ps). 